\title{
IDEOLOGICAL ANALYTICS
}

A STUDY OF EVOLVING WORLD VALUES

\author{
BY \\ PARIS ARNOPOULOS
}

CONCORDIA UNIVERSITY

MONTREAL

1990 
This study analyses ideologies in a comparative perspective. It does so by treating ideologies as normative mental systems interacting with environmental physical realities and following their evolution in the contemporary world. As a result, it exemplifies a systematic method of undertaking a diagnosis, anagnosis, and prognosis of the global ideological situation.

The study introduces a relevance tree reflecting the conceptual framework of ideological analytics. On this basis, it presents three levels of generality which contain the outline of a substantive analysis.

The examples given therein illustrate the model and could serve as prototypes for more detailed studies.

Finally, the study draws the main conclusions from the above generalizations by identifying the dialectical process of ideological dynamics. This process together with the non-linear development of social systems produce the macro-historical cycles of world evolution. Thus, the proposed hypothesis of dialectic Chaos can contribute to a better understanding of ideo-social change.

THEMATIC OUTLINE

1. IDEAL SYSTEMS:

1.1. CONCEPTUAL DEFINITION: Terms of Reference.

1.2. VALUE SPECTRA: Structural Preferences.

1.3. RELEVANCE TREE: Conceptual Framework.

2. IDEOLOGICAL MODELS: Substantive Analysis.

2.1. PRIMARY LEVEL: General Foundations.

2.2. SECONDARY LEVEL: Existential Reality.

2.3. TERTIARY LEVEL: Social Systems.

3. NORMATIVE DYNAMICS: General Deductions.

3.1. STRUCTURAL EVOLUTIONS: Multi-ideological Profiles.

3.2. STATISTICAL NORMS: Collective Distributions.

3.3. DIALECTICAL CHAOS: Non-linear Synthesis. 


\section{INTRODUCTION}

Although humans have always had ideals, ideology is a relatively recent concept. Represented by the three great contemporary movements (conservatism; liberalism; socialism) which originated in the modern western world, the term is a controversial one and means different things to different people. Similarly, attitudes towards ideologies vary from fanatical adherence in one to complete opposition against all of them.

Some scholars, who do not take such extreme views, simply see ideology as a historical phenomenon whose time had come with the Industrial Revolution and is now gone in the PostIndustrial world. Yet, these declarations about the end of ideology, like those about the death of God, may be greatly exaggerated!

Whether ideologies are ephemeral phenomena or chronic conditions depends on their relationship to the changing or persisting aspects of reality. Beyond that, it is a matter of definition if the particular conceptual label corresponds to a certain existential situation or not. The problem with such controversies, therefore, is both semantic and substantive, involving a play of words as well as a conflict of opinions.

In order to make a more scientific analysis: i.e. diagnosis, anagnosis and prognosis, on the state of ideology in the world, it is necessary to find an all-inclusive meta-ideological base which will serve as a common frame for our discourse. That such a base could be found was the starting hypothesis for this paper. What follows, then, is the search for and construction of a conceptual model whose main function is to view ideologies within a larger perspective in space and time.

The most useful schema for our purposes has been found in systems theory: which provides a sufficiently broad and flexible paradigm to contain such a complex and abstract subject. Regarding ideologies as symbolic systems allows for a better analysis of their structures and functions in comparison to other systems. Like all systems, ideologies share the same anatomic criteria which make for the proper conceptual compatibility. On that basis, this study will attempt an ideological analysis in generic terms.

The analysis will dissect the concept of ideology and look both inside and outside its boundaries. If we knowing what the components of this system are and how they operate we can clarify our understanding of this elusive subject and compare ideologies as to their similarities and differences. In this way, we shall be in a better position to evolve and evaluate them. 


\section{IDEAL SYSTEMS}

As sets of any elements, systems are groups of some interrelated components existing within a given environment. This minimal definition allows for anything to be defined as a system, provided it fulfills the following three conditions, i.e. that it possesses some distinct units; that these units have certain relations; and that they display identifiable boundaries.

Defined as such, systems may be divided into two main types: real (material) and ideal (mental). This basic dichotomy distinguishes concrete from abstract systems according to the nature of their make up; thus differentiating between things, organisms and people, on the one hand, and ideas, forms and concepts, on the other.

The two types are interrelated and interacting, thus forming a super-system containing both; much like the real and imaginary components of complex numbers. Obviously, physical reality affects human thoughts, just as much as putting our ideas into practice can change the environment. How this is done is, of course, part of the hoary mind-body problem, whose complete solution still escapes us.

This articles will not attempt so grandiose task, but simply look at the ideal side of the coin to see the profile of its changing outline. Understanding the statics and dynamics of ideological systems will enable us to improve our predictive capacity and thus foresee the probable ideological profile of the world at the turn of the millennium.

To do so, we shall begin by defining our subject-matter, thus drawing the boundaries of our universe of discourse. Then continue by specifying the relevant environment within which it can be situated. We will end this section by constructing a model framework within which ideologies may be compared and manipulated.

\subsection{CONCEPTUAL DEFINITION}

The term ideology was first coined by Destutt de Tracy (circa 1800), to designate a set of general ideas emanating from particular sensations. This definition was originally meant to substitute ideology for metaphysics, following Condillac's sensualist school which emphasized culture-specific theories, rather than objective-universal laws as guides to human behavior.

Of course, this etymological sense of the term has now been broadened to include any general system of values and beliefs, including religion and morality. Accordingly, ideology is an mental model which describes reality, explains man's place in it, and prescribes proper human behavior. By doing so, ideologies may be seen as normative as well as explanatory and cybernetic systems.

In this brief paper, we cannot look at the ideological system in all its manifold complexity. We are merely interested in the particular aspects which determine its relative position in time and space. For that reason, we define ideology simply as a system of normative perspectives.

This conceptual definition may be operationalized by translating ideologies as value preferences along an ordinal scale of priorities. This means that an ideology is the salient manifestation of a relative value position. By adhering to an ideology, one thereby adopts a preferential position regarding a central value selected among a relevant set of options.

Ideologies are usually recognized by labels ending in -ism; i.e. fasc-ism radical-ism, scient-ism. We shall follow this convention and relate ideologies with the dominant value 
associated with each one. For instance, liberalism is an ideology which implies an overwhelming preference for the value of liberty. Values will, therefore, serve as the common denominator of ideologies, thereby giving a comparative perspective to our ideological analysis.

\subsection{VALUE SPECTRA.}

As functions of ideologies, values are preferences for particular attractive properties, i.e. liberty, equality, fraternity. These properties are not absolute: that is to say they are neither present nor absent in their entirety. Rather, they have different degrees of a particular quality or quantity.

As such, they may be measured along a spectrum of increasing or decreasing strength. Although they may form discontinuous functions, most values are usually continuous along a gradated range between zero and one hundred per cent.as shown below.

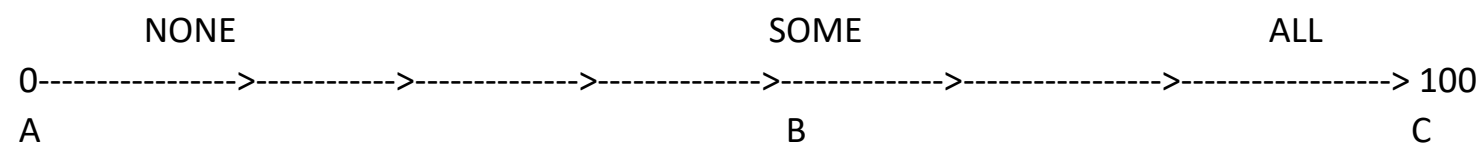

Different ideologies may be located along this continuum, depending on the degree of preference that each has for that particular value. In the above schema, ideology A has very low, ideology $B$ has medium, while ideology $C$ has very high regard. If, for instance, that value were equality, egalitarianism would be at the highest position, corresponding to ideology $C$.

In practice, values exist somewhere between the two ideal extremes, as partial attributes. Idealy defined, there is no such thing as any value which can exist on earth in its full measure. Values thus serve as ideals or goals to be sought after, but never attained completely.

Under the circumstances, it is better to consider only the middle segment of the value spectrum. This segment ranges somewhere between zero and $100 \%$, but is open ended in both directions, as shown below.

$\begin{array}{llll}\text { LEAST LESS SOME } & \text { MORE } \\ -<\leftarrow-->- & \text { MOST }\end{array}$

A crucial characteristic of values is that our preference for them does not change linearly. This means that there are diminishing returns associated with their increase. The more one has of a value, the less it is valued. Obviously, there is too much even of a good thing (e.g. a lot freedom is license). Usually, the desire for a value changes along with its availability, making scarce qualities more valuable than plentiful ones.

Similarly, the difficulty of attaining a value increases along with the value, so that the closer one gets to either extremity the more expensive each additional increment becomes. For that reason, values are generally attained in a modicum and resist both maximization and minimization.

Obviously, people vary in the amount of any value they can tolerate. Some want more of one and less of another at a particular time; or change their tolerance of a particular value 
at different times. This value variation within and between people is the reason of most personal and social conflicts, thus it is very important for our analysis.

Per definition, ideologies are sliding positions along the above value segments. As a desire to maximize a certain value, some ideology can be located at the high end of every continuum. In the same way, the position of a person relative to that ideology can also be located as either more or less between the maximum and minimum range of the corresponding value.

Accordingly, everybody has an ideological profile which is made up of the position one takes concerning the group of ideologies one likes best or hates most. Similar profiles may be traced for communities of people or nations, indicating average collective value preferences which characterise particular cultures or periods (cf. 3.2).

\subsection{RELEVANCE TREE.}

Having presented the major characteristics of ideologies and their underlying values, we are now ready to discuss the substantive criteria upon which they can be anchored. This will be done by outlining a general classification scheme that will serve as the overall framework of our concerns.

The thesis here is that the most significant ideological positions must be found in a relevance tree of fundamental values (see diagram below). These values relate to the central concerns of humanity towards which our preferences can be manifested and may be found at various levels of generality.

At the most basic level of human consciousness is the capacity to distinguish three primordial concepts: space; time; and being. Everything that we can perceive or conceive falls within this three-dimensional frame, so, it will be maintained as the foundation of our paradigm.

Above and beyond this basic level, are built various other ones which divide every dimension into further categories of increasing particularity. Thus, to take being, for example, its three main categories may be distinguished to be: nature, society, and humanity.

Each of these realms can be increasingly sub-divided into more specific components. Society, for instance, is traditionally composed of economic, political, and cultural sectors. This process of focusing on smaller and smaller areas can go on indefinitely until it hits the level of a given significance. In this brief study, we have chosen to deal with the three most basic levels and focus on the three central realms which interest us here. This scheme is shown in the following taxonomic diagram which outlines the nested relevance tree.

Of course, all conceptual distinctions are to a certain extent arbitrary. So the above categories do not necessarily exist outside the human mind; and what one mind can do, another can redo differently. This, however, need not bother us here; as long as we realize the relativity of human typologies.

The one proposed here has been found useful in conceiving and analyzing complex ideas, so it is presented below as a relevance tree containing the various branches of interest to this study. It is the major thesis here that major ideologies must take a normative position in all of these issue areas. So, our next step will be to present these positions in their comparative perspective. 


\section{IDEOLOGICAL MODELS}

On the basis of the above general considerations, we shall now give the specific framework of ideological statics and dynamics. The purpose here is to locate the major ideologies on the relevance tree introduced in the previous section. For that reason, we shall state the criteria used to situate ideologies relative to each other. These criteria will include the main parameters of reality and their interconnections at various levels.

Each of the nine areas of concentration will be dealt with by defining the polar opposites of each value in question and the range of positions within which an ideology could be located. Every area will, thus, contain a standard continuum upon which ideological selections can be made.

Once the static aspects have been determined, we move to the dynamics of ideological evolution. Obviously, ideologies do not remain in one location for ever. Human values change with time as well as place. This change can be indicated as a movement up (right) and down (left) of the value continuum which serves to support the ideology.

The movement of ideological positions can be followed historically by plotting their past performance. On that basis, certain trends may be discerned which if continued will indicate their future path. In the following analysis, we shall attempt to do that: i.e. diagnosis of present conditions; anagnosis of past histories; and prognosis of future trends.

The constraints of this article preclude an analysis in depth of our subject matter. What follows, then, is only some brief examples of the end results which such analysis can produce and not the detailed documentation which produced them. In any case, what is more important here is not the particular opinions of the author in each case study, but the overall methodology used in the general model.

\subsection{PRIMARY LEVEL}

As we perceive it, any holistic conceptual framework must contain three aspects of reality: spatial; temporal; and existential. This means that an ideology could be classified under the topology, chronology, or ontology of its subject-matter. Let us look into each of these realms.

The first value area concerns space and includes territoriality or geography. In this case, the central criterion is size, so the continuum ranges from the micro, through the meso, to the macroscopic, as shown below.

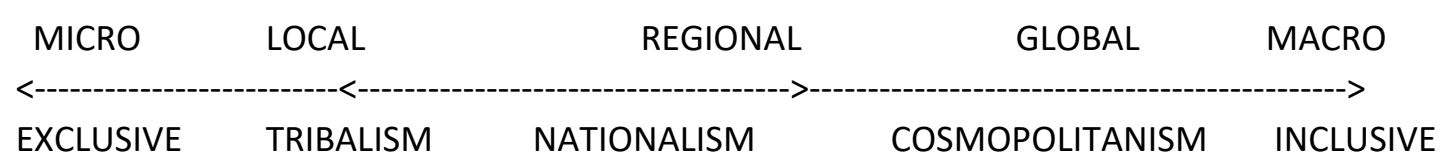

An ideology focuses on one of the above spatial levels whose scope becomes its highest value. In the modern world, the middle level predominates in most people's preferences, so Nationalism is the strongest contemporary ideology. In previous eras, the scope was much smaller and more exclusive, so the dominant ideology was infra-nationalistic. As science and technology broadens our horizons, however, Globalism becomes increasingly feasible as well as valuable and hence could replace Nationalism as the all-inclusive ideology of 
the world in the next century.

This brings us to the next area of concern which relates to time and includes temporality and history. The criterion here is the arrow of time which flies from the past, through the present, into the future, as shown below.

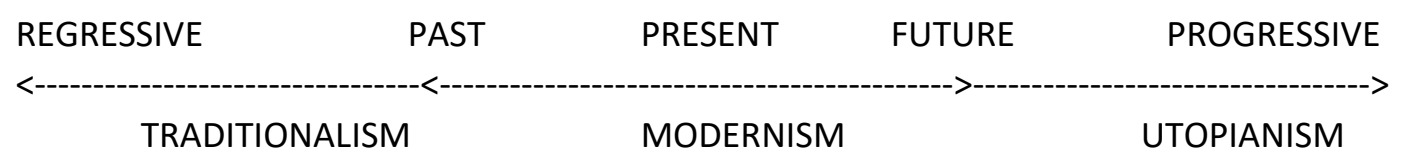

Ideologies that value the past and wish to preserve its traditions are obviously Conservative, as was the case throughout most of history. Modernism, however, brought forth a less pessimistic outlook and regards the present as an improvement upon the past. Optimists complete this range by seeing the future as the potential fulfilment of utopian ideologies.

Of course, there is a peripheral reaction to this, as to other heavy trends, So the world is experiencing a conservative revival by certain minorities which counters the modernist tendencies of the majority. These counter trends, however, are rearguard actions which delay but do not cancel the global tendency towards the future.

Finally, the third area of primary concern relates to existential reality and includes the substance and essence of being. The most useful criterion in this most complicated issue seems to be the degree of concreteness or abstractness in which various aspects of reality are viewed by the different ideologies shown below.

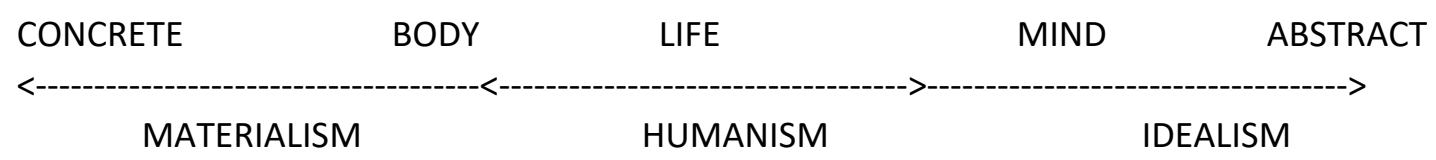

Ideologies that view reality as primarily physical are materialistic and oppose those idealistic ones that view it as mainly mental or spiritual. Between them is a more humanistic point of view which combines the two extremes in the medium of life.

Historically, it seems that the ideologies of dynamic societies swing back and forth between materialistic and idealistic periods. If progress leads to ideological stability, this pendulum effect may in the future settle down within the humanistic middle and thus avoid wild swings on either side.

It should be noted that in all these cases, the ideologies mentioned are only salient examples and do not exhaust the plethora of preferences along these scales. Whatever these preferences are, however, those who hold them cannot avoid taking a position in these three primary issues.

\subsection{SECONDARY LEVEL}

The importance of the existential realm outlined above requires further elaboration which will be undertaken now. Although, we could have done the same for all the realms, we limit the analysis in this one only; thus using it as an illustration of this method of subdivision.

In this second level of reality, we divide the existence in two antithetical areas (external and internal) connected by a third (intermediate) one. These areas may be 
considered as concentric circles from the largest (exosphere) to the smallest (endosphere).

Starting at the outermost boundary, which serves as the all-inclusive environment, we may subdivide it along the standard range used here. The criterion for this continuum will be the relation of existential entities to nature, as shown below.

\begin{tabular}{|c|c|c|}
\hline ARTIFICIAL & NATURAL & SUPERNATURAI \\
\hline TECHNOLOGISM & ECOLOGISM & THEOLOGISM \\
\hline
\end{tabular}

This scheme classifies the external world into three subdivisions, with nature occupying the middle position. At one end are the products of humanity and the secular ideologies which gives them primacy. At the other end is the terra incognita of magical and mystical concerns. Naturalism or Ecologism are intermediate positions between the known and unknown, physical and metaphysical, worlds of our environment.

In this area, historical evolution has swung the pendulum from Theism to Technism, but is now returning toward the centre. As technology creates more ecological problems, popular values are bound to move further toward Holism, as is expected to happen in the foreseeable future.

Closing in towards the innermost sphere of the internal world of each human being, we can discern a similar trend. This egosphere, as opposed to the ecosphere, may be equally subdivided on the criterion of human significance: i.e. the extent to which the internal world is dominant or subordinate to the external, as shown below.

\begin{tabular}{lcc}
\multicolumn{1}{c}{ PASSIVE } & AGONISTIC & ACTIVE \\
FATALISM & DETERMINISM
\end{tabular}

This scheme juxtaposes the belief that the fate of man is completely in the hands of the gods to the opposite conviction that man's free will can make us the masters of the universe. The former position leads to fatalistic ideologies and passivity, whereas the latter leads to arrogant ideologies and hubris. In between is the attitude that humanity is partly determined by external forces which it could try to overcome by continuous struggle.

The historical evolution here has been clearly from oriental Fatalism to occidental Voluntarism. However, as we reach the limits to growth, humanity becomes less optimistic about its unbounded capacity and is willing to accept some natural Determinism to limit its scope of action. The expectation, then is for a more balanced position which accepts some external constraints without giving up all internal initiatives.

This brings us to the intermediate world which connects the external and internal realms. It is well known as the social system and consists primarily of interpersonal relations. As semi-natural beings, humans are partly the producers and products of society, thus their behavior is determined by a combination of natural, personal and social factors.

Focusing on the social world, our chosen criterion is that of human affinity, i.e. the degree to which man is or should be part of the system. Along the standard continuum, this degree ranges between complete adherence and total isolation, as shown below. 


$\begin{array}{lcc}\text { CHAOTIC } & \text { MECHANIC } & \text { ORGANIC } \\ \text { INDIVIDUALISM } & \text { LEGALISM }\end{array}$

Individualist ideologies result from the belief that society is a very loose system (gesellschaft); whereas Collectivist ideologies think of it as a very tight community (gemeinschaft ). In the former, the individual is supreme; in the latter subordinate to the whole. Between these extremes is the legalistic view of the social contract, where the individual and the state share rights and duties.

The trend of modernity has shifted social evolution from the organic stability of primitive communities towards the chaotic dynamism of the Industrial Revolution. The Collectivist reaction to the Individualist extremes followed as a natural consequence in the 20th Century. This reaction, however, seems to have spent its force and the pendulum is swinging back towards the centre, where it might rest for a while as Constitutionalism becomes the dominant ideology of the next century.

This analysis of the existential realm allowed us to look into its three dimensions and see the detailed structure of their value criteria. The various ideological positions along these ranges may be combined to form ideological clusters, thus indicating the overall conceptual orientations towards these aspects of reality.

\subsection{TERTIARY LEVEL}

Because of the importance of the social system in our study, we shall now concentrate upon it and make a further analysis of its components. Taking a classical approach, we have grouped these components into three sectors: economic; cultural; and political. Each of them, relates to particular structures (market; family; state) and functions (distributive; reproductive; cybernetic), which we shall consider in turn.

We begin with the economy, since it is usually considered as the infrastructure of society. Economic ideologies may be classified according to their distributive criteria: i.e. on what basis and how extensive is their distribution of wealth. Distributive justice and property ownership are therefore central to economic values, as shown below.

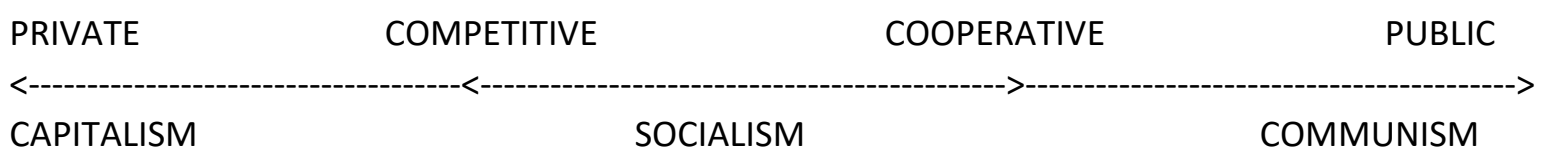

In this scheme, Capitalism's central value of private property is opposed to Communism's value of public good. Superimposed upon these values are the elitism of the former versus the egalitarianism of the latter. Between them lies Socialism, whose welfare state directs the accumulation and distribution of wealth.

As was the case with social evolution as a whole, economic trends have moved from primitive Communism to galloping Capitalism and back again to state Communism. Presently, the excesses of both sides are being denounced and a better equilibrium position is found in the social-market economy of post-industrial societies. 
The next item of our discussion is culture: the central sector of society. As such it has the most diverse structures and most complex functions of all social sectors. Nevertheless, the simplest criterion for classifying cultural ideologies is their degree of permissiveness. On this basis of cultural tolerance, the range is shown below.

\begin{tabular}{lcc}
\multicolumn{1}{c}{ RIGIDITY } & FLEXIBILITY & DIVERSITY \\
FUNDAMENTALISM & PRAGMATISM
\end{tabular}

One extreme of this continuum is occupied by the rigid ideologies of Fundamentalism, also shared by fanatical religions that allow no deviation from their orthodox beliefs. The other extreme is occupied by Pluralist ideologies of high tolerance for multiculturalism and co-existentialism. The mixed ideologies of the middle range are more pragmatic and utilitarian in their flexible response situational exigencies.

As social and geographical mobility increases, the historical pendulum is moving from cultural homogeneity to heterogeneity. The fundamentalist reaction in some societies attests to this dominant trend. Although some social systems may be fundamentalist in isolation, the world as a whole shall have to be pluralist or perish. Most likely, as the global system develops a cosmopolitan culture, the integrity of individual societies can only be maintained by a pragmatic policy of optimal flexibility.

Finally, since polity is considered as the superstructure of society, it is the last, but not least, sector of our presentation. Political ideals specify the best way to govern society; therefore, the relevant criterion here is the degree of state involvement in the life of individuals. In this respect, the different political ideologies would range as shown below.

$\begin{array}{lcc}\text { MINIMAL } & \text { LAISSEZ-FAIRE } & \text { INTERVENTION } \\ \text { CONSERVATISM } & \text { LIBERALISM } & \text { TOTALITARIANISM }\end{array}$

The continuum between minimal and maximal government is populated by many steps from the passive attitude of Conservative governments to the active involvement of Totalitarian states. In this range, Liberalism holds the middle ground by trying to provide sufficient individual freedom consistent with the necessary social control.

Political development has moved the ideological pendulum from minimal to maximal intervention within a few generations. As social systems become more complex, increasing political control seems as inevitable as it is necessary. The disadvantages of this trend, however, makes people resist it passionately. Thus, the most likely outcome is some kind of enlightened Liberalism, at least for the post-industrial countries in the turn of the century.

With these nine brief exposŽs, we complete the analytic outline of modern ideologies. Of course, we could have continued to even higher levels of specificity and looked at one or more of the social sectors (religion, education, legislation) for the anatomy of their parts. The above discussion, however, should suffice in establishing principles involved in this essay, so we leave further details to separate in-depth studies which could follow. 


\section{NORMATIVE DYNAMICS}

In order to undertake the above analysis, we used certain implicit assumptions which should now be made explicit. These assumptions involved the requirements of the analytic process as it was applied in this case. It is the form and content of these requirements that will be discussed here.

As we understand it, the necessary and sufficient conditions for the analysis of any system are: perceptual; conceptual; and operational. That is to say: a system must be accurately perceived on the basis of empirical facts; properly conceived on the basis of theoretical principles; and correctly operationalized on the basis of procedural rules. Thus, analysis combines data, models, and codes which are manipulated to produce its diagnosis, anagnosis, and prognosis of any given system.

In this last section, we will consider each of the three aspects and draw some theoretical generalizations concerning their ideological implications. These will be covered by discussions on three topics: structural; statistical and dialectical. In this way, we shall touch upon all the important factors involved in ideological analytics.

\subsection{STRUCTURAL EVOLUTIONS}

As they were depicted here, ideologies fell into one or another position of the general relavance tree. This picture, however, is a static simplification. The assigned position is neither exclusive nor exhaustive but only of central importance. It serves as a focus which gives the ideology a vantage point for its particular perspective.

These specialized normative positions are usually combined in practice fo form various clusters which make up the ideological profile of every person. The different permutations of the positions in the above nine criteria would constitute such personal profiles.

The ideological profiles of great minds have given their name to super-ideologies, such as Platonism, Thomism, and Marxism. These profiles are multi-dimensional because they group a number of values corresponding to all the variables we have outlined above. In these terms, Platonism, for instance, could be characterized as a combination of patriotism, utopianism, idealism, naturalism, determinism, collectivism, communism, fundamentalism, and totalitarianism.

Although some of these designations may be debatable and others added in greater detail, they suffice to outline in broad terms the essential values of these ideologies; at least so far as their social aspects are concerned. For religious ideologies, in particular, other categories will have to be specified, as necessary for the purpose of other studies.

All personal ideologies arise in a particular time and place, so they reflect the ideas and realities of that period and region. Although there is always a gap between ideological positions and existential conditions, the two are causally related. Ideas reflect facts, at the same time as they create or destroy them. This feedback between facts and values produces a dynamic spiral which alternates back and forth, thus shaping both in the image of each other.

For that reason, certain ideological combinations are more probable than others. Logical consistency and practical exigency make some clusters feasible or tenable in certain places and under certain conditions. Thus nationalism and progressivism have combined in many modern countries; just as certain conditions favored a Marxist domination in many parts of the world during most of the 20th Century.

Great religions are known for the extent of their scope in time and place. The greater the ideology the larger its spread and the longer its duration. Transcending time and space means rising above localism and relativism, thus approaching universality and eternity. Some great ideologies have 
dominated most of the world for long periods, in which case they became the distinctive weltanschauung of their historical eras.

The trends which we noted in the previous section make it quite probable that the world spirit at the turn of the century will most likely be a combination of internationalism, progressivism, humanism, ecologism, determinism, legalism, socialism, pluralism, and liberalism. Of course, this profile applies to the dominant global super-ideology. Individual countries, especially marginal ones, could deviate substantially from this mainline norm.

\subsection{STATISTICAL NORMS}

When a similar profile is shared by a large group of people, then we can speak of an ideological community, which is the characteristic of strong social systems. As part of a culture, ideologies contribute in keeping societies together or tearing them apart, depending on whether they are compatible or not.

Stable societies find most of their populations in the middle range of any statistical distribution. The dictum that the middle class is the pillar of society is therefore not far from the truth. This means that most people tend to cluster in mid-space between the ideological extremes of any value continuum. The will-known Bell curve would then apply to ideological distribution as well as to other normal attributes. As a result, our thesis here is that majorities seem to abhor extremes under normal conditions; because extreme positions are more difficult to attain and maintain (cf. 1.2).

On the contrary, critical situations are characterized by dichotomies in the normal statistical distributions. Confrontations arise when the two extremes of any range are populated more than the middle, thus increasing the potential of conflict. When this happens, if the system is not to be destroyed altogether, either a compromise will be worked out in the middle, or one side will win over the other.

In the former case, the system could return to the status quo ante. In the latter case, it shifts the framework to the winning side, so that what was a previous extreme now becomes the new middle. At the same time, what used to be the middle becomes one extreme position, while the opposite extreme is now occupied by the new ideology.

This process has been repeated in history, as new ideas and values replace the old. What used to be a radical ideology, i.e. liberalism, becomes quite middle of the road, from one century to the next. Similarly, what is taken for granted at one time (i.e. imperialism), disappears at another.

The dominant ideology of human societies throughout most of the world's history was a combination of tribalism, traditionalism, spiritualism, mysticism, fatalism, collectivism, socialism, fundamentalism, and conservatism. According to our analysis, this historical profile has been changing and the emerging one seems to converge towards the middle range of all dimensions.

Obviously, this may be seen as a middle-class optimistic prognosis, which depends on the continuation of certain trends and the deflection of others. As such it is a desirable utilitarian scenario because it prefers a more peaceful, humanitarian, and tolerant world than the present one. This can only come about if extremes of one kind or another are avoided and the bulk of the world's population converges towards the economic and political as well as the ideological center.

There is the possibility, however, that the movement reverses itself and what seemed to be a dead ideology, rises up again at another time or place. Ideologies, such as Fascism or Imperialism, may go through many revivals in history and could still rise to haunt us again, if conditions permit them anew. 
This is because, even if particular ideologies rise and fall, their underlying foundations remain the same. These are related to the constant parameters of physical and human nature, within which all variations must take place. Thus the shift of ideologies evolves in parallel with the continuity of permanence.

Whatever the dominant ideology of any particular period happens to be, there are always counter-ideologies to oppose it. Whatever, the majority at any time thinks, there are always minorities that think the contrary. Thus, there will always be some fascists, tribalists and fundamentalists, even in a democratic, global, pluralistic society.

\subsection{DIALECTICAL CHAOS}

The explanation for these contradictory phenomena is to be found in both the relative permanence of certain existential parameters and the evolution of others. Although, in the long run, nothing remains the same, the rate of change varies with the level of reality. Thus, superstructures change much faster than infrastructures and nature much slower than culture.

Much of social change, taking place within natural stability, is non-linear. Periods of stability or slow and gradual change, alternate with periods of flux and revolution. The transition from one to the other condition may be caused by a small random fluctuation or an unintentional action which snowballs into a major crisis and even deterministic chaos.

What is important for our purposes here is to emphasize that every idea generates its opposite and every value has a counter-value; just as to every action there is a reaction and for every position there is bound to be an opposition. Human minds as well as social systems create such dichotomies and fluctuate between them. The extremes of these theses and antitheses may be eventually mediated or resolved by a syntheses of both into an intermediate value position.

This dialectical process may be said to be the general conceptual principle which determines ideological evolution, as it does mental and social processes. Dialectics may be said to be at the center of political compromise as well as natural confrontation. The contradictory nature of many facts and values, thus, gives ideologies their controversial character and infuses history with its cyclic progressions.

This characteristic also makes it impossible for all values to be maximized at once. Every idea contains the seeds of its own destruction which flower as that idea is carried to extremes. Moreover, since most values are mutually exclusive, the more of one means the less of another (i.e. liberty is inversely proportional to security). Utopian visions of perfect societies, where there is plenty of everything are, therefore, as feasible as perpetual motion machines.

The recent discoveries of non-linearity in complex systems together with the dialecticity of opposite concepts has produced a powerful tool in explaining reality. In that sense, the new theory of chaos combines with the old theory of dialectics to reflect more accurately the complex dynamics of natural, social and ideological development.

Our thesis here is that ideologies, as all complex systems, behave according to the combined principles of dialectical chaos. This means that they follow certain rules at one level, while appearing to flaunt then at another. Thus, apparent contradictions reflect fundamental unities of the same reality and chaotic behavior is only so in the eyes of the beholder. 


\section{CONCLUSION}

In closing this brief paper we shall reiterate our major theses and then draw the appropriate conclusions. First of all, ideologies are an innate feature of human mentality because they provide a descriptive, interpretive and prescriptive model of reality; something that is necessary for rational behavior.

The different value priorities of each human being, however, ensure the existence of a multitude of opposing ideological preferences. This opposition creates inter and intra-personal conflicts which are often of a zero-sum nature: one ideology's gain is another's loss. The comparative scheme devised in the relevance tree clearly shows this inverse proportionality between all value dualities.

The contradictions resulting from this situation may be resolved dialectically, in which case new ideologies emerge along with their accompanying social change. The non-linearity of historical evolution attests to this process which may be coined as dialectichaos.

Accordingly, the shifting ideological positions in social systems tend to swing, both diatopically and diachronically, between dialectical extremes and then settle closer to the middle. The natural tendency of most people to converge towards the statistical middle provides normative standards which act as centers of gravity or strange attractors for ideological as well as physiological systems.

On the basis of this theory, the various ideological profiles of the contemporary world will tend to converge to their median position as a normal process of historical evolution. After the unstable critical periods of the 20th century, the surprise-free prognosis is for a settling down of the mainstream ideological profile of the world. Thus, barring some unpredictable catastrophe, the evolving global culture will parallel an emerging Gaian ideology on the threshold of the next century. 


\section{BACKGROUND BIBLIOGRAPHY}

Arnopoulos, P.J. (Ed). Prospects for Peace. Gamma, Montreal, 1986.

Bell, D. The End of Ideology. Macmillan, N.Y. 1961.

Bertalanffy, L. General Systems Theory. Braziller, N.Y. 1975.

Corbett, P. Ideologies. Hutchinson, London, 1965.

Csanyi, V. Evolutionary Systems \& Society. Duke U.P. Durham, 1989.

Ebenstein, W. Today's Isms. Prentice-Hall, N.J. 1970.

Gyorgy, A \& Blackwood, G. Ideologies in World Affairs. Ginn, N.Y. 1968.

Lane, R. Political Ideology. Macmillan, N.Y. 1967.

Laszlo, E. Evolution. Shambhala, Boston, 1987.

Love, N. Dogmas \& Dreams. Chatham House, N.J. 1989.

McCullough, H. (Ed). Political Ideologies. Thompson, Toronto, 1990.

Plamenatz, J. Ideology. Macmillan, N.Y. 1971.

Ritt, M.A. Dictionary of Modern Political Ideologies. Manchester UP.1990.

Sargent, S. Contemporary Political Ideologies. Dorsey, Chicago, 1987.

Teune, H. \& Mlinar, Z. The Developmental Logic of Social Systems.

Sage, B.H. 1978. 